\title{
7 Impact of a Patient Navigator Program on Hospital-Based and Outpatient Utilization Over 180 Days in a Safety-Net Health System
}

\author{
Richard B. Balaban, $M D^{1,2}$, Fang Zhang, $P h D^{2,3}$, Catherine E. Vialle-Valentin, $M D^{2,3}$, \\ Alison A. Galbraith, MD, MPH ${ }^{2,3}$, Marguerite E. Burns, $\mathrm{PhD}^{4}$, Marc R. Larochelle, MD, $\mathrm{MPH}^{5}$, and \\ Dennis Ross-Degnan, $S C D^{2,3}$
}

\begin{abstract}
'Somerville Hospital Primary Care, Cambridge Health Alliance, Somerville, MA, USA; ${ }^{2}$ Harvard Medical School, Boston, MA, USA; ${ }^{3} \mathrm{Harvard}$ Pilgrim Health Care Institute, Boston, MA, USA; ${ }^{4}$ University of Wisconsin, Madison, WI, USA; ${ }^{5}$ Boston University School of Medicine, Boston, MA, USA.
\end{abstract}

BACKGROUND: With emerging global payment structures, medical systems need to understand longer-term impacts of care transition strategies.

OBJECTIVE: To determine the effect of a care transition program using patient navigators (PNs) on health service utilization among high-risk safety-net patients over a 180-day period.

DESIGN: Randomized controlled trial conducted October 2011 through April 2013.

PARTICIPANTS: Patients admitted to the general medicine service with $\geq 1$ readmission risk factor: (1) age $\geq 60$;

(2) in-network inpatient admission within prior 6 months;

(3) index length of stay $\geq 3$ days; or (4) admission diagnosis of heart failure or (5) chronic obstructive pulmonary disease. The analytic sample included 739 intervention patients, 1182 controls.

INTERVENTIONS: Through hospital visits and 30 days of post-discharge telephone outreach, PNs provided coaching and assistance with medications, appointments, transportation, communication with primary care, and self-care.

MAIN MEASURES: Primary outcomes: (1) hospital-based utilization, a composite of ED visits and hospital admissions; (2) hospital admissions; (3) ED visits; and (4) outpatient visits. We evaluated outcomes following an index discharge, stratified by patient age ( $\geq 60$ and $<60$ years), using a 180-day time frame divided into six 30-day periods.

KEY RESULTS: The PN program produced starkly different outcomes by patient age. Among older PN patients, hospital-based utilization was consistently lower than controls, producing an $18.7 \%$ cumulative decrease at 180 days ( $p=0.038)$; outpatient visits increased in the critical first 30-day period $(p=0.006)$. Among younger PN patients, hospital-based utilization was $31.7 \%(p=0.038)$ higher at 180 days, largely reflecting sharply higher utilization in the initial 30 days $(p=0.002)$, with non-significant changes thereafter; outpatient visits experienced no significant changes.

\section{NIHTrial Reaistration ClinicalTrials, gov identifier: NCT01619098}

Electronic supplementary material The online version of this article (doi:10.1007/s11606-017-4074-2) contains supplementary material, which is available to authorized users

Received November 21, 2016

Revised March 31, 2017

Accepted April 14, 2017

Published online May 18, 2017
CONCLUSIONS: A PN program serving high-risk safetynet patients differentially impacted patients based on age, and among younger patients, outcomes varied over time. Our findings highlight the importance for future research to evaluate care transition programs among different subpopulations and over longer time periods.

KEY WORDS: care transitions; continuity of care; health care delivery; patient safety; underserved populations.

J Gen Intern Med 32(9):981-9

DOI: $10.1007 / \mathrm{s} 11606-017-4074-2$

() Society of General Internal Medicine 2017

\section{INTRODUCTION}

The 30-day readmission rate has become the benchmark quality measure used to evaluate hospital-to-home transitional care; it has been adopted by the National Quality Forum ${ }^{1}$ and is publicly reported by the Centers for Medicare and Medicaid Services. ${ }^{2}$ The focus on 30-day readmissions gained initial traction in studies linking the quality of transitional care and early hospital readmissions; ${ }^{3,4}$ more recently, randomized trials have demonstrated that some early readmissions are preventable with improved inpatient and post-discharge care $^{5-7}$ The current widespread use of the 30-day readmission rate reflects its value in assessing the short-term impact of readmission reduction interventions in specific populations of patients. $^{8}$

Focusing solely on 30-day outcomes, however, has limitations. First, the quality benefits of short-term interventions such as improved medication management, higher rates of outpatient follow-up, and reduced nearterm readmissions may not be sustained over time. To truly improve patient care, readmissions must not simply be deferred beyond a 30-day accounting window, but rather long-term decreases in hospital-based utilization and better connection to outpatient care must result. Second, most 30-day readmission analyses consider only one discharge per patient, minimizing the effect of high utilizers who make an oversized contribution to longterm hospital-based utilization. For example, in Massachusetts, just $7 \%$ of frequently hospitalized patients were 
responsible for $25 \%$ of all medicine or surgery discharges over a 1 -year period. ${ }^{9}$ Thus, for payers (public and private) or hospital administrators responding to intensified pressures to control long-term medical costs, fully accounting for all utilization within and beyond the 30day post-discharge period is essential.

We conducted a randomized controlled trial using hospitalbased community health workers (CHWs) as patient navigators (PNs) to provide assistance for 30 days to high-risk general medicine patients transitioning from hospital to home. In prior work, we used a standard analytic approach to evaluate the effects of the PN intervention on 30-day readmission rates after an index discharge. ${ }^{10}$ However, the PN program design was distinctive in that PNs provided transitional support to patients after each qualifying medicine service admission over the entire study period; patients admitted multiple times received multiple "doses" of the 30-day PN intervention. Thus, the PN program was intentionally structured as a "reallife" care transition program might function.

In this paper, we analyze intervention impacts on all hospital-based care (emergency department [ED] visits and hospital admissions) and outpatient appointments over a 180 day period. We also report results in the six successive 30-day periods within this 180-day time frame in order to examine changes in observed effects over time. While 30-day measures assess an intervention's ability to prevent near-term readmissions, the 180-day perspective evaluates whether an intervention, when implemented as a sustained readmission reduction program, can benefit a defined patient population over a prolonged period.

\section{METHODS}

\section{Study Setting, Participants, and Randomization}

Our study setting and design have been described previously. ${ }^{10}$ We targeted hospitalized patients within Cambridge Health Alliance (CHA), an academic public safety-net system with an ethnically and linguistically diverse population. CHA is an integrated healthcare provider comprising two hospitals, three EDs, and 10 community health centers.

Enrollment took place from October 2011 through April 2013. Eligible community-dwelling patients with a primary care provider (PCP) within CHA were randomized to the intervention or control group during their first CHA medicine service observation or inpatient admission. Eligible patients had at least one of five readmission risk factors, identified in previous studies ${ }^{11-15}$ and in CHA historical data: (a) age $\geq 60$; (b) admission to CHA's medicine, surgery, or psychiatry service within the past 6 months; (c) index length of stay $(\mathrm{LOS}) \geq 3$ days; (d) admission diagnosis of heart failure or (e) chronic obstructive pulmonary disease. In contrast to most care transition studies, our eligible population included nonEnglish speakers and patients who were homeless, left against medical advice, had a diagnosis of dementia, or were discharged to a skilled nursing facility (SNF).

Our randomization scheme allowed PNs to specify the number of intervention patients to enroll each day in order to maintain a daily census of 30-35 patients per full-time PN. A computer algorithm randomly assigned the $\mathrm{PN}$-specified number of eligible patients to the intervention group and the remaining eligibles to controls. Patient assignment to intervention or control remained constant throughout the study period.

After randomization, discharge from the medicine service to home or to SNF was considered a "qualifying" discharge, i.e., intervention patients were eligible to receive PN services. Patients transferred from medicine to another service (e.g., psychiatry or surgery), a non-network hospital, long-term care, or law enforcement, or who were discharged from nonmedicine services (e.g., surgery or psychiatry), were ineligible to receive PN support; however, all such discharges were included in our hospital-based utilization outcome measure. The trial ended after the PNs had completed their pre-specified period of effort.

Our study was powered to evaluate 30-day readmissions following an index qualifying discharge. ${ }^{10}$ As previously described, the stratified analysis, revised early in the study, reflects the strikingly different risk profiles of patients over and under age 60 , potentially affecting response to the intervention. ${ }^{10}$

The institutional review boards of Cambridge Health Alliance and Harvard Pilgrim Health Care approved the study as quality improvement and low risk to patients, waiving the requirement for signed consent.

\section{Control and Intervention Groups}

Control patients received CHA's usual inpatient, transitional, and outpatient care. Intervention patients received enhanced transitional support over each qualifying 30-day postdischarge period. During the hospitalization, PNs visited with intervention patients and families to establish rapport, describe the PN program, assess post-discharge needs, highlight the importance of post-discharge engagement, and schedule the first post-discharge PN call. After discharge, via weekly telephone contacts, the PNs helped arrange transportation to outpatient appointments, addressed barriers to obtaining or taking medications, identified patients' concerns and facilitated communication with PCP offices, reassessed patients' home care needs and made connections to services, assisted with health insurance problems, and supported patient self-management. Intervention patients received the same PN services after each subsequent qualifying discharge.

\section{Study Data and Outcome Measures}

Study data were extracted from the CHA EMR for 180 days after the index discharge. There were four primary prespecified outcome measures, all tracking exclusively innetwork data: (1) hospital-based utilization, a composite of 
ED visits and hospital admissions (observation or full) to any service (medical, surgical, or psychiatric); (2) hospital admissions; (3) ED visits; and (4) outpatient visits. In a prior analysis, the PN intervention produced markedly different shortterm outcomes for patients over and under age $60^{10}-$ we continue using the same stratification by patient age to evaluate longer-term effects. A secondary exploratory analysis evaluates the effect of behavioral health $(\mathrm{BH})$ conditions (mental health or substance use disorder) on our primary outcomes.

We performed an intention-to-treat analysis; all randomized patients were included except those who died during their index admission. We censored patients if their 180-day follow-up periods extended beyond the end of the trial. With no way of ascertaining deaths in the community or patients lost to follow-up, we included all study patients for the 180 day duration following their index discharge or until the end of the trial.

To adjust for residual imbalances in characteristics between randomized groups, we estimated a propensity score that reflects the likelihood of assignment to the PN group using demographic and baseline clinical characteristics including gender; race (white/non-white); English speaking; number of ED visits and admissions in the 6 months preceding the index admission; history of substance abuse, BH diagnoses, or dementia; Charlson score; LOS over 2 days, and index admission hospital. We used the resulting propensity scores to create inverse probability weights, used in all subsequent analyses. All analyses are stratified by age $\geq 60$ or $<60$ years. We compared baseline characteristics of intervention and control groups using chi-square tests, $t$-tests, and non-parametric tests. ${ }^{16}$

We used population-level general estimating equation (GEE) models with a negative binomial distribution and inverse probability weights to model outcome rates; the negative binomial distribution ${ }^{17,18}$ was selected to adjust for overdispersion of the data. ${ }^{19}$ Because some patients were censored, our models also adjust for number of days contributed in each 30-day period by individual patients using offsets. ${ }^{19}$

We estimated the differences in rates in each period by calculating the adjusted differences between intervention and control groups and their confidence intervals, setting all other variables in the models to their average values. To display cumulative differences over the 180-day period, we summed the 30-day adjusted differences between groups and used the delta method to calculate the confidence intervals of the summed differences. All analyses were conducted using Stata version 12 software (StataCorp LP, College Station, TX, USA).

\section{RESULTS}

\section{Participant Characteristics}

Of 12,488 first admissions to a medicine service during the study period, 1937 met study eligibility criteria. After excluding 16 deaths during the index admission, the analytic sample included 739 intervention patients and 1182 controls (Fig. 1). Of these, 597 (80.8\%) PN patients and 771 (65.2\%) control patients provided the full 180 days of follow-up data. The intervention group's higher rate of 180-day follow-up is primarily due to the greater number of controls enrolled near the trial end, when the available PN effort had declined.

The overall study population was diverse in race and language, mostly publicly insured, with a high burden of $\mathrm{BH}$ conditions. Randomization elicited comparable intervention and control patients in both age groups, with exceptions as noted (Table 1 and eTable 1-online). Over the 180-day followup period, 482 patients received the PN intervention only once, 132 patients twice, and 66 patients three or more times following qualifying discharges (data not shown).

\section{Outcomes}

Among older PN patients, aged $\geq 60$ years, there was a cumulative reduction of 0.21 hospital-based encounters per patient at 180 days, a decrease of $18.7 \%(p=0.038$; Table 2$)$. Hospital-based encounters were lower in the PN group than in controls in every 30-day period (Fig. 2 and eTable 2-online). The cumulative difference in hospital admissions at 180 days was not significant $(p=0.188)$, although admissions were non-significantly lower in the older PN group in four of the six 30-day periods. The cumulative difference in ED visits trended towards significance $(p=0.09)$, with fewer ED visits in the older PN group in all six 30-day periods. The cumulative difference in outpatient visits was not significant $(p=0.197)$, although outpatient visits in the older PN group were significantly higher in the 30 days following the index discharge $(p=0.006)$ and non-significantly higher during all subsequent 30-day periods.

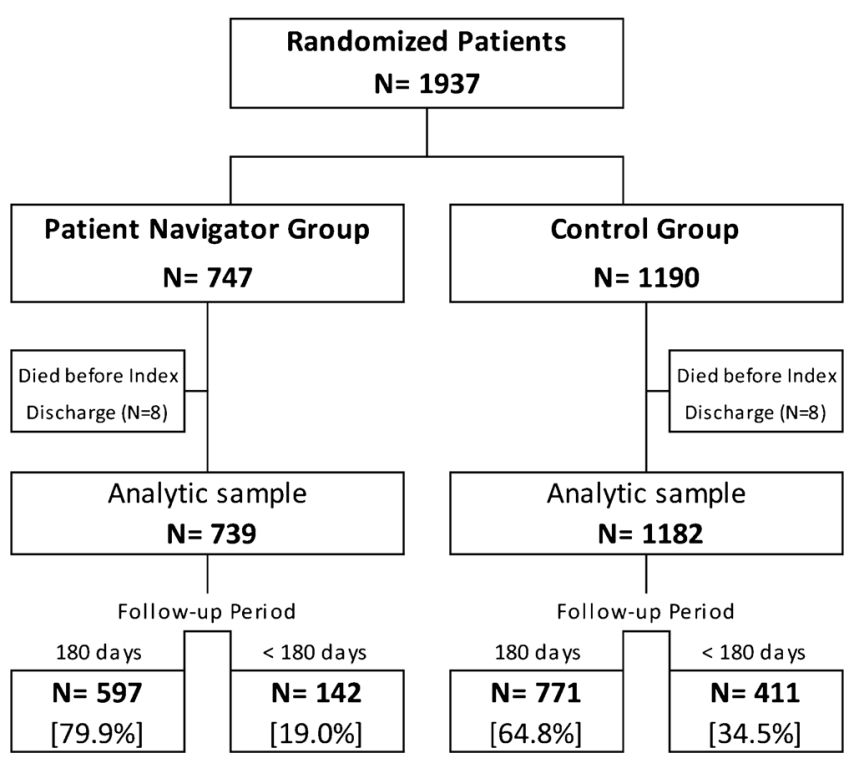

Figure 1 Flow diagram of patient participation. 
Table 1 Baseline Characteristics of the Study Sample by Intervention Group and Age Group

\begin{tabular}{|c|c|c|c|c|c|c|}
\hline \multirow[t]{3}{*}{ Characteristics of randomized patients } & \multicolumn{3}{|c|}{ Age $60+$ years } & \multicolumn{3}{|c|}{ Age $<60$ years } \\
\hline & \multirow{2}{*}{$\frac{P N}{(N=545)}$} & \multirow{2}{*}{$\frac{\text { Control }}{(N=788)}$} & \multirow[b]{2}{*}{$p$-value } & \multirow{2}{*}{$\frac{P N}{(N=194)}$} & \multirow{2}{*}{$\frac{\text { Control }}{(N=394)}$} & \multirow[b]{2}{*}{$p$-value } \\
\hline & & & & & & \\
\hline Age at index discharge, mean (SD) & $74.5(9.8)$ & $75.1(9.4)$ & 0.2786 & $46.2(9.6)$ & $45.7(10.8)$ & 0.5906 \\
\hline Female, $n(\%)$ & $315(57.8)$ & $483(61.3)$ & 0.2005 & $95(49)$ & $205(52)$ & 0.485 \\
\hline \multicolumn{3}{|l|}{ Language, $n(\%)$} & \multirow[t]{5}{*}{0.6388} & & & \multirow[t]{5}{*}{0.4153} \\
\hline English & $330(60.6)$ & $480(60.9)$ & & $153(78.9)$ & $302(76.6)$ & \\
\hline Portuguese & $88(16.1)$ & 109 (13.8) & & $19(9.8)$ & $30(7.6)$ & \\
\hline Spanish & $40(7.3)$ & $64(8.1)$ & & $12(6.2)$ & $38(9.6)$ & \\
\hline \multirow{2}{*}{\multicolumn{3}{|c|}{ Race, $n(\%)$}} & & $10(5.2)$ & $24(6.1)$ & \\
\hline & & & \multirow[t]{5}{*}{0.6346} & & & \multirow[t]{5}{*}{0.8829} \\
\hline White & $349(64)$ & $485(61.5)$ & & $113(58.2)$ & $241(61.2)$ & \\
\hline Black & $81(14.9)$ & $129(16.4)$ & & $25(12.9)$ & $44(11.2)$ & \\
\hline Hispanic & $54(9.9)$ & $91(11.5)$ & & $41(21.1)$ & $82(20.8)$ & \\
\hline Other & $61(11.2)$ & $83(10.5)$ & & $15(7.7)$ & $27(6.9)$ & \\
\hline \multicolumn{3}{|l|}{ Health insurance type, $n(\%)$} & \multirow[t]{6}{*}{0.8204} & & & \multirow[t]{6}{*}{0.6302} \\
\hline Medicare & $240(44)$ & $351(44.5)$ & & $5(2.6)$ & $13(3.3)$ & \\
\hline Medicaid & $91(16.7)$ & $131(16.6)$ & & $95(49)$ & $185(47)$ & \\
\hline Dual Medicare \& Medicaid & $127(23.3)$ & $195(24.7)$ & & $48(24.7)$ & $83(21.1)$ & \\
\hline Health Safety Net* & $58(10.6)$ & $79(10)$ & & 27 (13.9) & $61(15.5)$ & \\
\hline Commercial/other & $29(5.3)$ & $32(4.1)$ & & $19(9.8)$ & $52(13.2)$ & \\
\hline Weighted Charlson score, mean (SD) & $1.4(1.5)$ & $1.2(1.5)$ & 0.1129 & $0.8(1.4)$ & $0.8(1.4)$ & 0.9344 \\
\hline \multicolumn{7}{|l|}{ Chronic behavioral health issues } \\
\hline Psychiatric dx before or at index, $n(\%)$ & $242(44.4)$ & $370(47)$ & 0.3582 & $133(68.6)$ & $272(69)$ & 0.9061 \\
\hline Substance abuse (SA) before or at index, $n(\%)$ & $96(17.6)$ & $166(21.1)$ & 0.119 & $110(56.7)$ & $242(61.4)$ & 0.2722 \\
\hline Psychiatric dx or SA before or at index, $n(\%)$ & $280(51.4)$ & $433(54.9)$ & 0.1985 & $151(77.8)$ & $315(79.9)$ & 0.5522 \\
\hline Dementia, $n(\%)$ & $57(10.5)$ & $85(10.8)$ & 0.8486 & $27(13.9)$ & $56(14.2)$ & 0.9229 \\
\hline \multirow{2}{*}{\multicolumn{7}{|c|}{$\begin{array}{l}\text { Qualifying risks of readmission } \\
\text { Age } 60+\text { years, } n(\%)\end{array}$}} \\
\hline & & & & & & \\
\hline Index admission LOS $\geq 3$ days, $n(\%)$ & $256(47)$ & $402(51)$ & 0.1467 & $116(59.8)$ & $283(71.8)$ & 0.0033 \\
\hline Admission(s) at CHA, past 6 months, $n(\%)$ & $87(16)$ & $122(15.5)$ & 0.8123 & $127(65.5)$ & $197(50)$ & 0.0004 \\
\hline Admissions at CHA, past 6 months, mean (SD) & $0.2(0.7)$ & $0.2(0.7)$ & 0.9656 & $1.1(1.3)$ & $0.8(1.2)$ & 0.0019 \\
\hline $\mathrm{COPD}^{\$}, n(\%)$ & $43(7.9)$ & $41(5.2)$ & 0.0472 & $15(7.7)$ & $28(7.1)$ & 0.7842 \\
\hline Heart failure ${ }^{\S}, n(\%)$ & $41(7.5)$ & $47(6)$ & 0.2599 & $7(3.6)$ & $7(1.8)$ & 0.1708 \\
\hline Index length of stay, mean (SD) & $3.1(2.5)$ & $3.6(4)$ & 0.0011 & $4.2(4)$ & $5(4.9)$ & 0.0499 \\
\hline Discharge against medical advice, $n(\%)$ & $4(0.7)$ & $5(0.6)$ & 0.8275 & $8(4.1)$ & $11(2.8)$ & 0.3905 \\
\hline CHA hospital-based care, past 6 months, $n(\%)$ & $204(37.4)$ & $299(37.9)$ & 0.8493 & $163(84)$ & $292(74.1)$ & 0.0069 \\
\hline CHA hospital-based care, past 6 months, mean (SD) & $0.8(1.5)$ & $0.8(1.5)$ & 0.7751 & $3.2(4.1)$ & $2.3(3.5)$ & 0.0124 \\
\hline ED visits, past 6 months, $n(\%)$ & $154(28.3)$ & $242(30.7)$ & 0.3351 & $114(58.8)$ & $225(57.1)$ & 0.7023 \\
\hline ED visits, past 6 months, mean (SD) & $0.5(1.3)$ & $0.5(1.2)$ & 0.7125 & $2.1(3.7)$ & $1.5(2.9)$ & 0.0765 \\
\hline Outpatient visits, previous 30 days, $n(\%)$ & $356(65.3)$ & $498(63.2)$ & 0.4271 & $128(66)$ & $261(66.2)$ & 0.9492 \\
\hline Outpatient visits, previous 30 days, mean (SD) & $1.3(1.4)$ & $1.3(1.5)$ & 0.8076 & $1.5(1.7)$ & $1.6(2.1)$ & 0.3781 \\
\hline
\end{tabular}

COPD chronic obstructive pulmonary disease

*Health Safety Net is a Massachusetts state program for uninsured patients

†Based on ICD-9 discharge codes 290-302.9 and 306-319

$\$$ Based on ICD-9 discharge codes 303-305.93

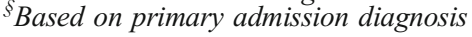

Table 2 Cumulative Differences in Rates of Outcomes (PN - Control) at 180 Days, by Age Group and Diagnosis of Behavioral Health Conditions*

\begin{tabular}{|c|c|c|c|c|c|c|}
\hline & \multicolumn{3}{|l|}{ Age 60+ years } & \multicolumn{3}{|l|}{ Age $<60$ years } \\
\hline & $\begin{array}{l}\text { Difference } \\
\text { [95\% CI] }\end{array}$ & $\begin{array}{l}\% \\
\text { Change }\end{array}$ & $p$-value & $\begin{array}{l}\text { Difference } \\
\text { [95\% CI] }\end{array}$ & $\%$ Change & $p$-value \\
\hline All patients & $n=1333$ & & & $n=588$ & & \\
\hline Hospital-based utilization & $-0.21[-0.41,-0.01]$ & $-18.7 \%$ & 0.038 & $0.79[0.14,1.45]$ & $31.7 \%$ & 0.017 \\
\hline Admissions & $-0.07[-0.18,0.03]$ & $-12.6 \%$ & 0.188 & $0.29[0.04,0.55]$ & $41.0 \%$ & 0.024 \\
\hline Outpatient visits & $0.44[-0.23,1.11]$ & $6.8 \%$ & 0.197 & $0.85[-0.46,2.17]$ & $10.6 \%$ & 0.202 \\
\hline Patients with behavioral Health Issues & $n=713$ & & & $n=466$ & & \\
\hline Hospital-based utilization & $-0.24[-0.51,0.02]$ & $-18.9 \%$ & 0.069 & $0.91[0.12,1.69]$ & $32.6 \%$ & 0.024 \\
\hline Admissions & $-0.02[-0.19,0.14]$ & $-3.7 \%$ & 0.776 & $0.35[0.04,0.66]$ & $45.4 \%$ & 0.025 \\
\hline Outpatient visits & $0.38[-0.59,1.35]$ & $6.1 \%$ & 0.438 & $0.67[-0.82,2.16]$ & $8.2 \%$ & 0.381 \\
\hline Patients without behavioral Health Issues & $n=620$ & & & $n=122$ & & \\
\hline Hospital-based utilization & $-0.13[-0.40,0.13]$ & $-15.4 \%$ & 0.318 & $0.18[-0.59,0.95]$ & $13.5 \%$ & 0.649 \\
\hline Admissions & $-0.10[-0.22,0.03]$ & $-21.1 \%$ & 0.141 & $0.01[-0.34,0.37]$ & $2.6 \%$ & 0.943 \\
\hline Outpatient visits & $0.62[-0.29,1.52]$ & $9.2 \%$ & 0.182 & $0.53[-1.76,2.83]$ & $6.6 \%$ & 0.648 \\
\hline
\end{tabular}

*Adjusted for gender, language, race, comorbidities, chronic behavioral health issues, pre-randomization CHA admissions and ED visits, index admission length of stay, and hospital (Whidden vs. Cambridge). Cumulative differences represent the sum of the difference PN minus control in each 30-day period cumulated over the 180-day study window 


\section{Hospital-based Utilization (ED Visits \& Admissions)}
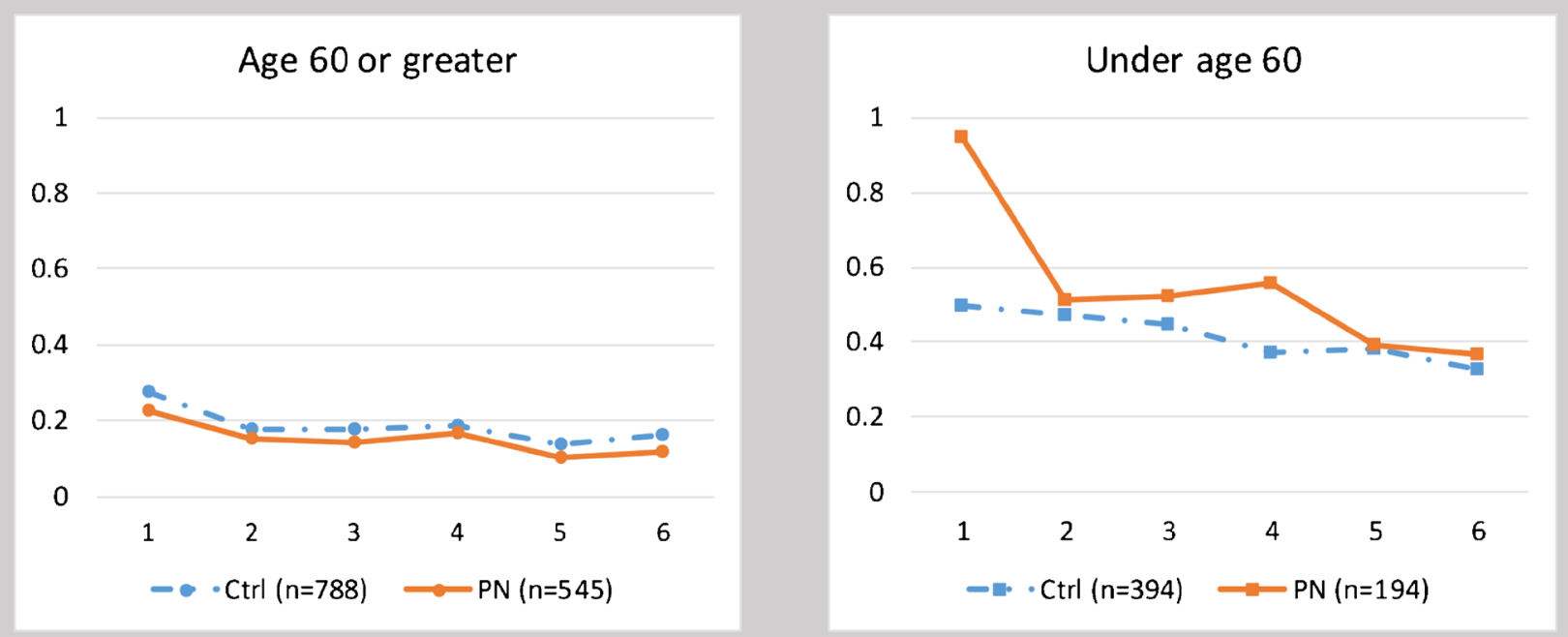

\section{Admissions}
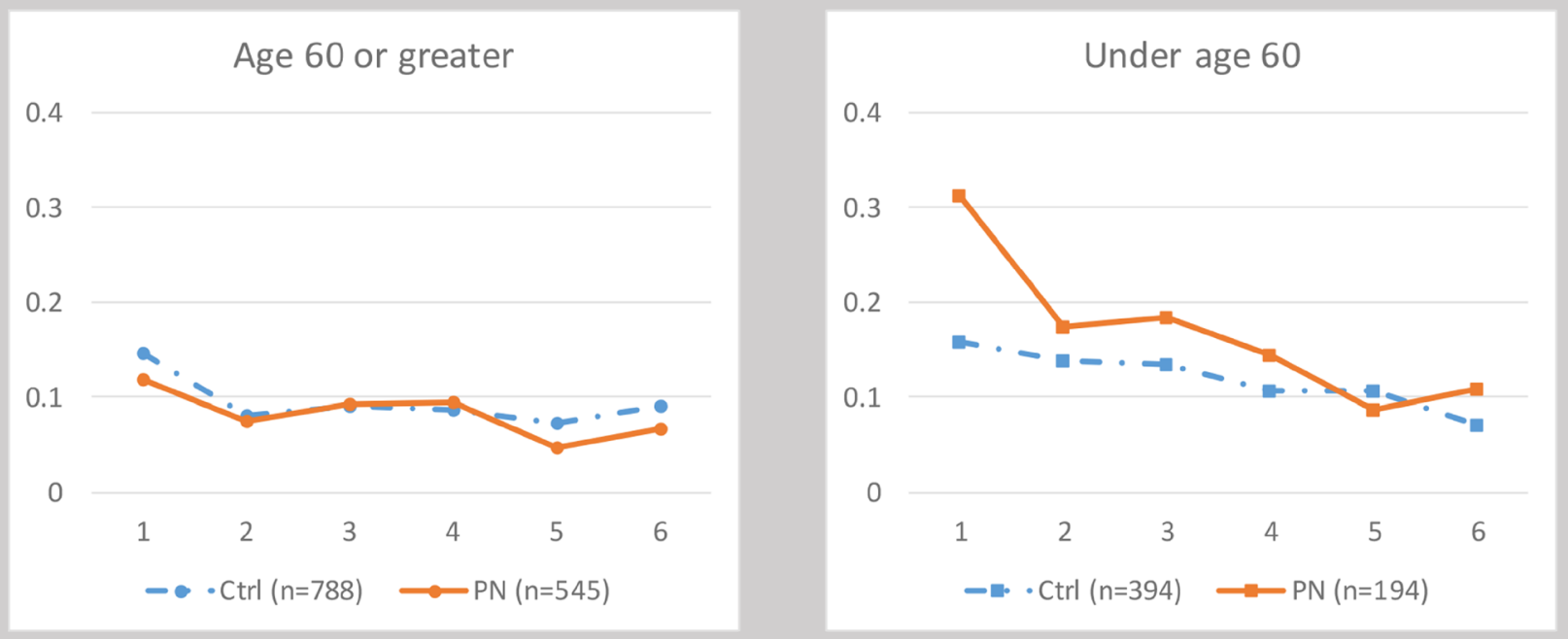

\section{Outpatient Visits}
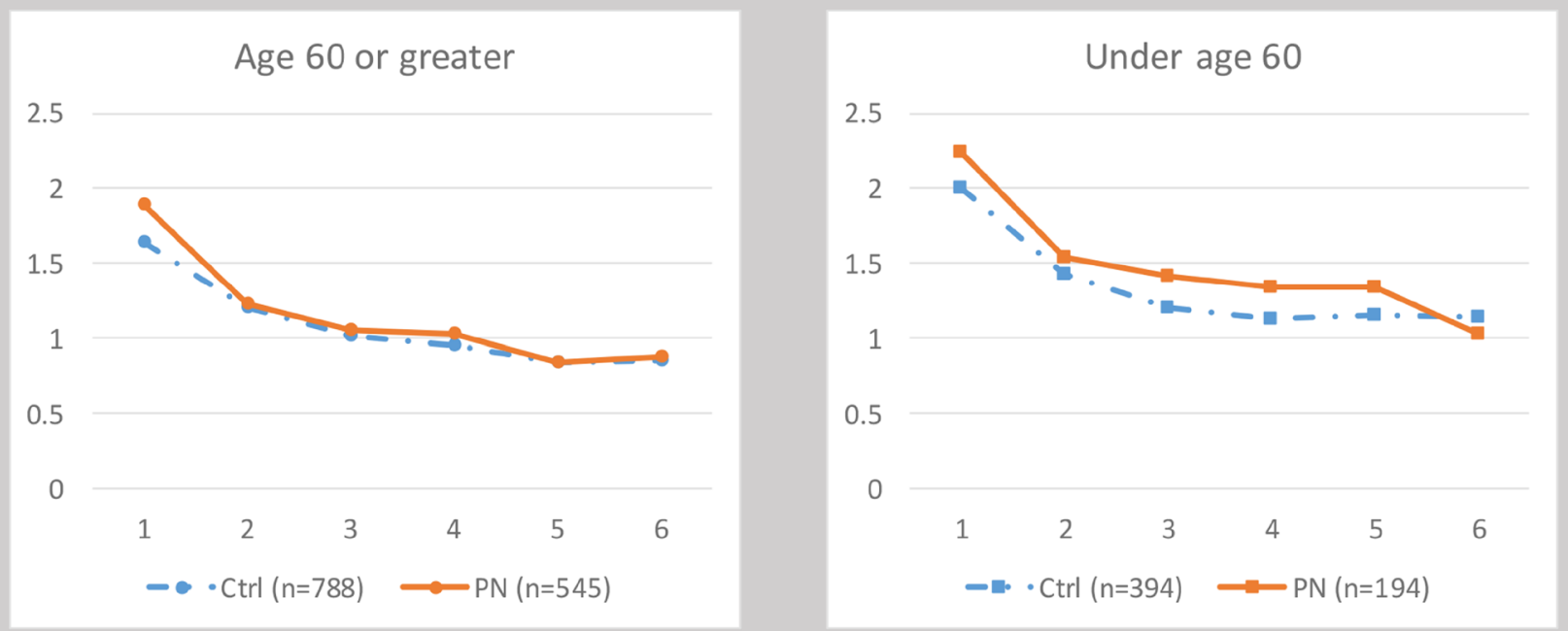

Figure 2 Rates of 30-day outcomes-sum of events per person per 30-day period. 
Among younger PN patients, aged $<60$ years, there was a cumulative increase of 0.79 hospital-based encounters $(31.7 \%, p=0.017)$ per patient at 180 days, largely reflecting sharply higher utilization $(p=0.002)$ in the PN group in the initial 30-day period, with smaller, non-significant increases in subsequent 30-day periods. The cumulative difference in hospital admissions at 180 days was an increase of $41.0 \%$ ( $p=0.024)$; again, largely reflecting the significantly higher admissions $(p=0.002)$ in the younger PN group in the initial 30-day period, with smaller non-significant increases observed in four of the subsequent five 30-day periods. The cumulative difference in ED visits was not significant ( $p=0.146$ ), although the PN group had increased visits in five of the six 30-day periods. The cumulative difference in outpatient visits was also not significant $(p=0.202)$.

To better understand the paradoxical increases in hospitalbased utilization among younger patients, we performed hypothesis-generating analyses. Among younger patients with baseline $\mathrm{BH}$ issues, there was a cumulative increase in hospital-based encounters of $32.6 \%(p=0.024)$ at 180 days, largely reflecting the significantly higher utilization $(p=0.006)$ in the PN group in the initial 30-day period (Fig. 3 and eTable 2-online). In contrast, among younger patients without $\mathrm{BH}$ issues, hospital-based utilization was similar between the intervention and control groups throughout the 180-day follow-up period $(p=0.649)$. Among older patients, the presence of baseline $\mathrm{BH}$ issues had no consistent relationship to the intervention effect on hospital-based encounters.

Finally, we tested the sensitivity of our results to differential length of follow-up by limiting the sample to subjects with 180 days of follow-up; the findings were consistent with our main results (data not shown).

\section{DISCUSSION}

The PN program used hospital-based CHWs to improve transitional care for high-risk general medicine patients after an index admission and all subsequent medicine service readmissions. The program employs well-accepted strategies, including initial in-person PN-patient interaction, and close collaboration between PNs and patients' primary care teams. ${ }^{20}$ Our extended 180-day analytic time frame provides a robust assessment of programmatic impact on utilization, including a comprehensive measure of hospital-based utilization that tallies ED visits and hospital admissions to any service, thereby fully reflecting the contribution of high utilizers. The 180day findings, similar to our previously reported 30-day results, ${ }^{10}$ are differentiated by patient age. However, the extended analytic window allows firmer conclusions about program effects in the older population, while raising new questions about the younger cohort. Additionally, our age-stratified analysis demonstrates how varying intervention responses may emerge.
Among older patients, the program was associated with a statistically significant decline in hospital-based utilization at 180 days, with decreases in each 30-day post-index period. For every five patients assigned to the PN program, one hospital-based encounter was prevented over 180 days. While decreases in ED visits were the main driver of reduced hospital-based care, smaller decreases in hospitalizations also contributed to the significant reduction of the composite measure.

These results reflect the combined effect of multiple PN doses (for patients having more than one medicine service readmission) and the potential sustained effects of any earlier dose. The PNs successfully directed older patients back to outpatient providers during the critical 30-day post-discharge period, which may have averted the need for further hospitalbased care. In summary, the durability of the response to the PN intervention, an $18.7 \%$ decrease in hospital-based utilization over 180 days, may make the use of low-cost CHWs a compelling option for those seeking to reduce avoidable highcost medical care in older high-risk patients.

Among younger patients, the PN program produced significant increases in hospital-based utilization; for every five patients assigned to the PN program, two additional hospitalbased encounters occurred over 180 days. Such paradoxical findings are not unique; care transition interventions have resulted in readmission increases of up to $96 \%$ among highrisk inpatients, including those on a psychiatric-substance abuse unit, ${ }^{21}$ medically complex Veterans Affairs patients, ${ }^{22}$ younger general medicine patients, ${ }^{23}$ and patients with heart failure. $^{24}$

However, our longer-term analysis revealed that large initial increases in utilization among younger patients were followed in later 30-day periods by more modest increases, with essentially no differences from controls in the final 60 days. There are several possible explanations for this pattern of decreasing utilization. First, the PN program is designed to increase access to care, which may lead to increased utilization to address unmet health needs. ${ }^{25}$ Once needs are met, utilization may diminish and stabilize. ${ }^{25,26}$ Alternatively, utilization may have decreased because PNs were unable to sustain patient engagement.

Second, while the PN program sought to increase timely post-discharge outpatient care, such increases were not seen among younger patients. Interestingly, many younger patients, particularly those with significant $\mathrm{BH}$ issues, use the $\mathrm{ED}$ as a source of their primary care, ${ }^{27-29}$ often based on the belief that hospital-based care is of higher quality ${ }^{30}$; notably, $38.3 \%$ of younger PN patients had two or more ED visits in the 6 months prior to their index admission. Thus, as the PN guides younger patients back to their primary site of care, some return to the $\mathrm{ED}$, where readmission becomes more likely. Third, the higher rate of prior admissions among younger $\mathrm{PN}$-assigned patients might indicate that they were at higher risk for hospitalization. Finally, our exploratory analyses indicate that active $\mathrm{BH}$ issues may have been the driving force in increased hospital-based 


\section{Hospital-based Utilization (ED Visits \& Admissions)}
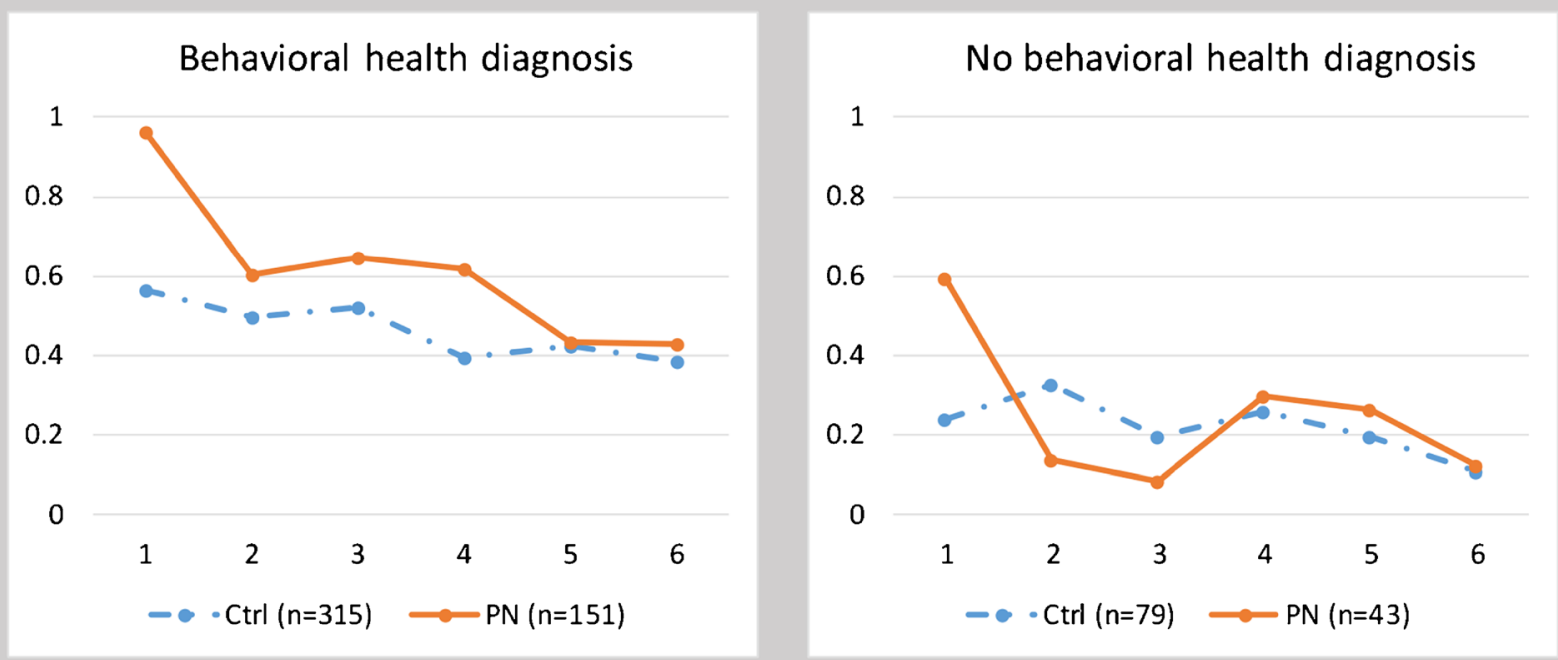

\section{Admissions}
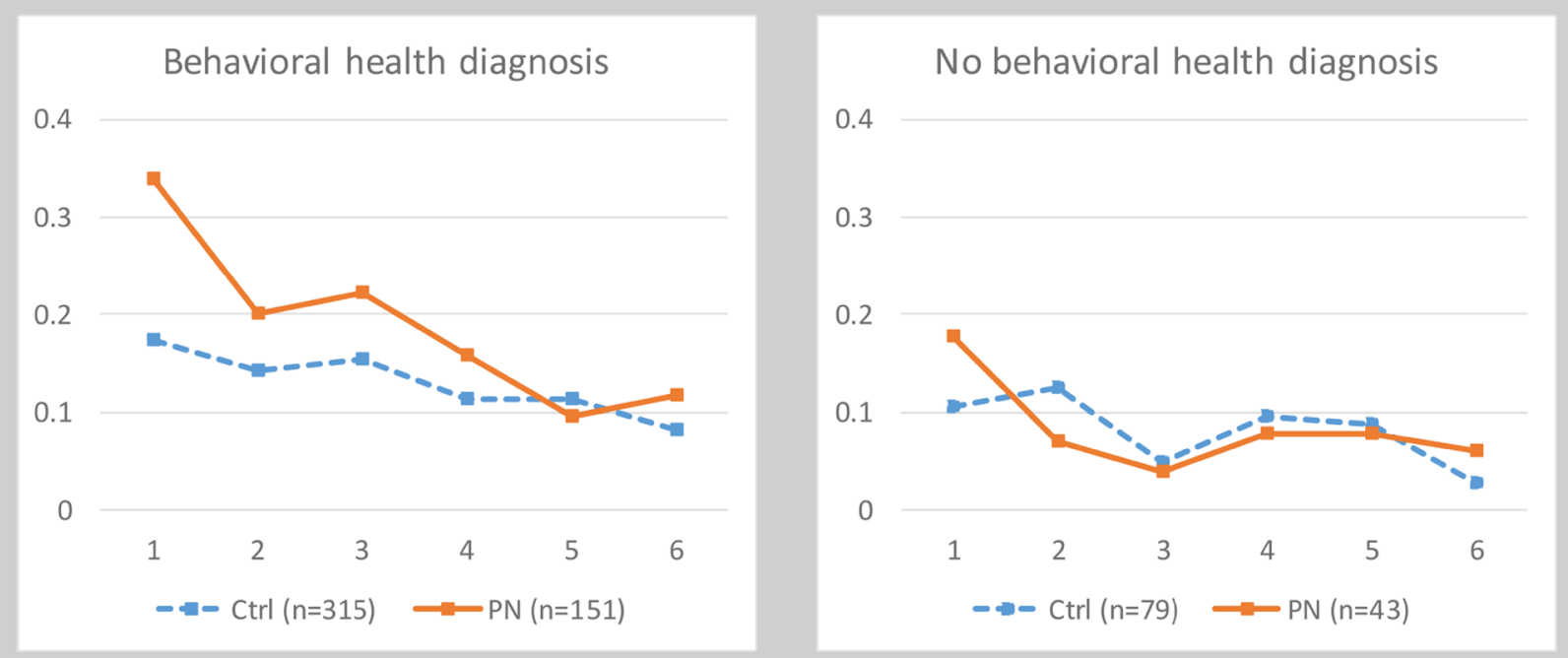

\section{Outpatient Visits}
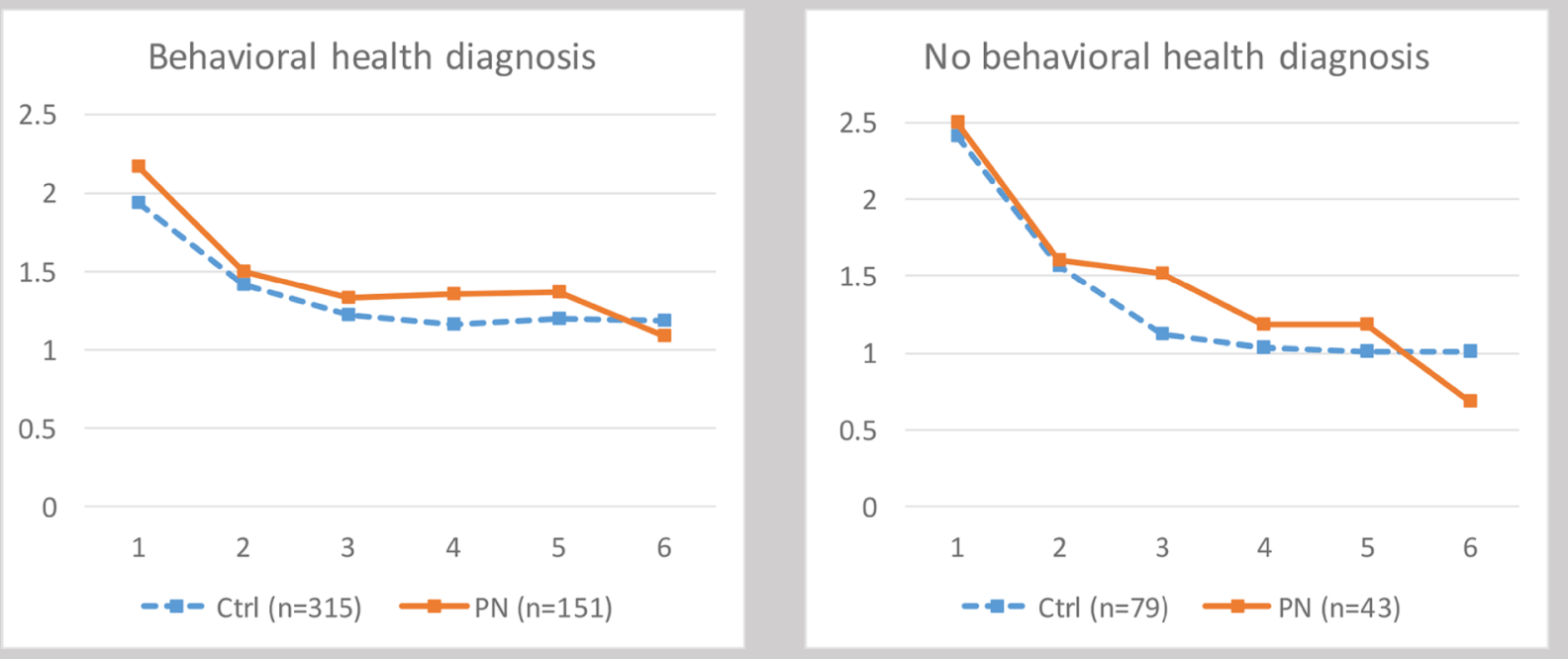

Figure 3 Rates of 30-day outcomes in patients aged $<60$ years - sum of events per person per 30-day period. 
utilization among younger PN patients. With limited mental health resources, including restricted hours of outpatient service, hospital-based care may be the only expedient option. ${ }^{31}$

Our analysis has several limitations. First, only medicine service discharges received navigation. If patients had received navigation after discharges from any service, the magnitude of change in hospital utilization might have been greater. Second, the 180-day time frame gives an important longer-term vantage point. While results in the older population are stable over time, results for younger patients are not, suggesting that examining a time frame longer than 180 days might provide further clarity, especially for patients with complex psychosocial issues. Third, lacking access to claims data, we captured only in-network admissions. We anticipated that out-ofnetwork admissions, estimated to be $20 \%,{ }^{32}$ would occur equally between intervention and control groups. However, a positive experience with a PN might encourage patients to preferentially select a CHA hospital for subsequent care; if this is true, our results might understate reductions in hospital-based utilization among older patients, while overstating increases in the younger population. Fourth, our analysis cannot provide definitive explanations for the divergent findings between age groups. We believe that the PN program helped patients receive needed post-discharge care-outpatient care for older patients and hospital-based care for younger patients. It is unlikely that PN activities led to unnecessary admissions for younger patients, since RNs and MDs made all medical decisions. However, alternative outpatient services specifically addressing $\mathrm{BH}$ issues might have prevented some hospital-based utilization among younger patients. Fifth, older PN patients had a shorter index LOS than controls, raising the possibility that hospitalists were comfortable discharging PN patients sooner, knowing that additional home support was available. If earlier discharge increased the likelihood of readmission, then decreased readmissions among older patients may have been understated. Finally, our study occurred within a single health system serving a largely publicly insured safety-net population; further validation would be required before generalizing to other settings.

\section{CONCLUSION}

A 180-day utilization analysis provides an important complement to 30-day readmission measures. While the 30day measure quantifies the effectiveness of a care transition intervention, our 180-day measure seeks to quantify the effectiveness of a care transition program. Older patients demonstrated consistent decreases in hospital-based utilization over 180 days, coupled with initial increases in outpatient care; younger patients showed large initial increases in hospital-based utilization that moderated over time, without changes in outpatient care. The dynamic nature of our results over the 180-day period, especially among younger patients, highlights the importance of examining longer-term trends. Indeed, with the proliferation of global payment programs, there will be great interest in future research that quantifies longer-term outcomes in order to identify high-quality cost-effective transitional care strategies.

Corresponding Author: Richard B. Balaban, MD; Somerville Hospital Primary CareCambridge Health Alliance, Somerville, MA, USA (e-mail: rbalaban@challiance.org).

\section{Author Contribution}

Sharon Touw assisted with the preparation of the manuscript.

\section{Compliance with Ethical Standards:}

Funders: This study was funded by the Agency for Healthcare Research and Quality (1R01HS020628-01A1). A pilot study was funded by Partners Community Healthcare, Inc., and the Department of Population Medicine at Harvard Medical School.

Prior Presentations: None.

Conflict of Interest: The authors declare that they do not have a conflict of interest.

\section{REFERENCES}

1. National Quality Forum. Measuring Performance. 2017. http://www. qualityforum.org/Measuring_Performance/Measuring_Performance. aspx. Accessed Jan 10, 2017.

2. Hospital Compare. 2016. https://www.cms.gov/medicare/quality-initiatives-patient-assessment-instruments / hospitalqualityinits / hospitalcompare.html. Accessed Jan 10, 2017.

3. Benbassat J, Taragin M. Hospital readmissions as a measure of quality of health care: advantages and limitations. Arch Intern Med. 2000;160(8):1074-1081.

4. Ashton CM, Del Junco DJ, Souchek J, Wray NP, Mansyur CL. The association between the quality of inpatient care and early readmission: a meta-analysis of the evidence. Med Care. 1997;35(10):1044-1059.

5. Hansen LO, Young RS, Hinami K, Leung A, Williams MV. Interventions to reduce 30-day rehospitalization: a systematic review. Ann Intern Med. 2011;155(8):520-528.

6. Leppin AL, Gionfriddo MR, Kessler M, et al. Preventing 30-day hospital readmissions: a systematic review and meta-analysis of randomized trials. JAMA Intern Med. 2014;174(7):1095-1107.

7. Kansagara D, Chiovaro JC, Kagen D, et al. So many options, where do we start? An overview of the care transitions literature. J Hosp Med. 2016;11(3):221-230.

8. Horwitz L, Partovian C, Lin Z, et al. Hospital-Wide (All-Condition) 30Day Risk-Standardized Readmission Measure. Centers for Medicare and Medicaid Services. 2011;2011:59.

9. Center for Health Information and Informatics. Hospital-Wide Adult AllPayer Readmissions in Massachusetts: 2011-2013. 2015. http://www. chiamass.gov/assets/docs/r/pubs/15/CHIA-Readmissions-ReportJune-2015.pdf. Accessed April 13, 2017.

10. Balaban R, Galbraith A, Burns M, Vialle-Valentin C, Larochelle $\mathbf{M}$, Ross-Degnan D. A patient navigator intervention to reduce hospital readmissions among high-risk safety-net patients: a randomized controlled trial. J Gen Intern Med. 2015;30(7):907-915.

11. Smith DM, Giobbie-Hurder A, Weinberger M, et al. Predicting nonelective hospital readmissions: a multi-site study. Department of Veterans Affairs Cooperative Study Group on Primary Care and Readmissions. J Clin Epidemiol. 2000;53(11):1113-1118.

12. Billings $\mathbf{J}$, Dixon $\mathbf{J}$, Mijanovich $\mathbf{T}$, Wennberg $\mathbf{D}$. Case finding for patients at risk of readmission to hospital: development of algorithm to identify high risk patients. BMJ. 2006;333(7563):327. 
13. Jencks SF, Williams MV, Coleman EA. Rehospitalizations among patients in the Medicare fee-for-service program. N Engl J Med. 2009;360(14):1418-1428.

14. Amarasingham R, Moore BJ, Tabak YP, et al. An automated model to identify heart failure patients at risk for 30-day readmission or death using electronic medical record data. Med Care. 2010;48(11):981-988.

15. Hasan O, Meltzer DO, Shaykevich SA, et al. Hospital readmission in general medicine patients: a prediction model. J Gen Intern Med. 2010;25(3):211-219.

16. Corder GW, Foreman DI. Nonparametric Statistics: An Introduction. Nonparametric Statistics for Non-Statisticians: A Step-by-Step Approach. New York: John Wiley \& Sons; 2009:1-11

17. Diggle PHP, Liang K-Y, Zeger S. Analysis of Longitudinal Data. Oxford: Oxford University Press; 2013.

18. Liang K-Y, Zeger S. Longitudinal data analysis using generalized linear models. Biometrika. 1986;73:13-22.

19. McCullagh P, Nelder J. Generalized Linear Models. 2nd ed. Boca Raton: Chapman \& Hall; 1989.

20. Peikes D, Chen A, Schore J, Brown R. Effects of care coordination on hospitalization, quality of care, and health care expenditures among Medicare beneficiaries: 15 randomized trials. JAMA. 2009;301(6):603618.

21. Tracy K, Burton M, Nich C, Rounsaville B. Utilizing peer mentorship to engage high recidivism substance-abusing patients in treatment. Am J Drug Alcohol Abuse. 2011;37(6):525-531

22. Weinberger M, Oddone EZ, Henderson WG. Does increased access to primary care reduce hospital readmissions? Veterans Affairs Cooperative Study Group on Primary Care and Hospital Readmission. N Engl J Med. 1996;334(22):1441-1447.

23. Einstadter D, Cebul RD, Franta PR. Effect of a nurse case manager on postdischarge follow-up. J Gen Intern Med. 1996;11(11):684-688.
24. Mudge A, Denaro C, Scott I, Bennett C, Hickey A, Jones MA. The paradox of readmission: effect of a quality improvement program in hospitalized patients with heart failure. J Hosp Med. 2010;5(3): 148-153.

25. Tobias C, Levinson J, Simon L. Reinventing the HMO: The Next Generation of Medicaid Managed Care. Center for Health Care Strategies, Inc. 2005. http://www.chcs.org/resource/reinventing-the-hmo-medicaid-managed-care-for-members-with-complex-needs/. Accessed April $13,2017$.

26. Finkelstein A, Taubman S, Wright B, et al. The Oregon Health Insurance Experiment: Evidence from the First Year. $\mathrm{Q} \mathrm{J}$ Econ. 2012;127(3):1057-1126.

27. Weissman JS, Stern RS, Epstein AM. The impact of patient socioeconomic status and other social factors on readmission: a prospective study in four Massachusetts hospitals. Inquiry. 1994;31(2):163-172.

28. Byrne M, Murphy AW, Plunkett PK, McGee HM, Murray A, Bury G. Frequent attenders to an emergency department: a study of primary health care use, medical profile, and psychosocial characteristics. Ann Emerg Med. 2003;41(3):309-318.

29. Tang N, Stein J, Hsia RY, Maselli JH, Gonzales R. Trends and characteristics of US emergency department visits, 1997-2007. JAMA. 2010;304(6):664-670.

30. Kangovi S, Barg FK, Carter T, Long JA, Shannon R, Grande D. Understanding why patients of low socioeconomic status prefer hospitals over ambulatory care. Health Affairs. 2013;32(7):1196-1203.

31. Baicker K, Taubman SL, Allen HL, et al. The Oregon experiment-effects of Medicaid on clinical outcomes. N Engl J Med. 2013;368(18):1713-1722.

32. Kind AJ, Bartels C, Mell MW, Mullahy J, Smith M. For-profit hospital status and rehospitalizations at different hospitals: an analysis of Medicare data. Ann Intern Med. 2010;153(11):718-727. 